$$
|A A-S \cup B--95-21|
$$

\title{
SUMNER ASSOCIATES
}

100 Cienega, Suite $D$

Santa Fe, New Mexico 87501

Telephone: 505 984-8041 FAX 505 984-3251

\section{FINAL REPORT \\ 0412J0004-3G \\ TASK 19}




\section{DISCLAIMER}

Portions of this document may be illegible in electronic image products. Images are produced from the best available original document. 


\section{Instrument Design Program Progress}

P. A. Seeger

Sumner Associates, B.O.A. 0412J0004-3G, Task 19

May 1 - Sept. 30, 1995

The goals of the task for this time period were met, and the resulting tools were used to obtain significant results in a number additional studies. Six presentations will be published in the Proceedings of ICANS-XIII, the $13^{\text {th }}$ Meeting of the International Collaboration on Advanced Neutron Sources, Paul Scherrer Institut, Villigen, Switzerland, October 11-14, 1995.

1. The principle task in this time period was to make the neutron optics Monte Carlo library MCLIB as general as possible, and to collaborate in the construction of an objectoriented user interface to make it easy for relatively untrained users to use the codes to test neutron instrument designs. As of Sept. 30, the interface could be used to define all element types necessary to study small-angle diffractometers or surface reflectometers. The prototype interface was presented at ICANS-XIII:

"A User-Friendly, Graphical Interface for the Monte Carlo Neutron Optics Code MCLIB,"

T. Thelliez, L. Daemen, P. A. Seeger, and R. P. Hjelm.

2. New features were added to the library to support simulation of additional instruments. New capabilities include a crystal monochromator, powder diffraction sample, Ikeda-Carpentrer pulse shapes, single-crystal filter, vertical and counter-rotating choppers, and isotropic scatterers. "The library was fully documented and presented at ICANS-XIII:

"The MCLIB Library: Monte Carlo Simulation of Neutron Scattering Instruments," P. A. Seeger.

3. Four instruments are being studied and optimized using the library. Three of these (the exception being the High Resolution Powder Diffractometer) were presented at ICANS-XIII:

"Small-Angle Scattering Instruments on a $1 \mathrm{MW}$ Long Pulse Spallation Source," G. A. Olah, R. P. Hjelm, and P. A. Seeger.

"The Performance of Reflectometers at a Continuous Wave and Pulsed-Neutron Sources," M. R. Fitzsimmons and R. Pynn.

"Monte Carlo Study of the Performance of a Time-of-Flight Multichopper Spectrometer," L. L. Daemen, J. Eckert, R. Pynn, and P. A. Seeger.

4. I. Ines of communication and data transfer from the team performing target-moderator calculations were established, to facilitate feedback from instrument optimizations to the target design. A library of "standard" source functions for the simulations has been created, and also presented at ICANS-XIII:

"Performance of Long Pulse Source Reference 'Target-Moderator-Reflector Configurations," E. J. Pitcher, G. J. Russell, P. A. Seeger, and P. D. Ferguson. 


\title{
Monte Carlo Simulation of Neutron Scattering at CW and LPSS
}

\author{
P. A. Seeger \\ Sumner Associates and LANSCE
}

A library of Monte Carlo subroutines has been developed for the purpose of design of neutron scattering instruments. Using small-angle scattering as an example, the philosophy and structure of the library will be described and the programs will be used to compare instruments at a variety of neutron sources.

\section{Statement of the Problem}

We want to know how an instrument copied from D11 at the Institut Laue-Langevin (ILL) reactor would perform on a long-pulse spallation source (LPSS). We choose to compare at a specific configuration of D11, namely $10.5 \mathrm{~m}$ collimation length and $10.5 \mathrm{~m}$ sample-to-detector distance, for a total instrument length of $21 \mathrm{~m}$ as shown in fig. 1. Instead of simulating the losses of the guides and velocity selector at D11, we use the measured flux at the sample [1], doubled to represent improvement with the new velocity selector "Costanze" [2]. The wavelength distribution is assumed to be triangular with $12 \%$ FWHM. To make the collimation identical for the two cases, we stop the LPSS moderator down to the size of the D11 guide exit, $30 \times 50 \mathrm{~mm}^{2}$, and fix the sample diameter at $10 \mathrm{~mm}$. The same detector is also assumed for both cases.

The LPSS instrument uses choppers to define a bandwidth, and then achieves wavelength resolution (which may be much narrower than the bandwidth) by measuring the time of flight (TOF) of each neutron from the moderator to the detector. The total neutron flux on sample will be higher because the bandwidth does not need to be limited by the resolution; a simple measure of TOF Gain is the ratio of the bandwidth to the resolution width. (More precisely, it is the integrated flux on sample over the full bandwidth divided by the integrated flux over one resolution width.) The maximum achievable bandwidth is inversely proportional to the instrument length and to the pulse repetition rate. For $21 \mathrm{~m}$ and $60 \mathrm{~Hz}$, it is $3.1 \AA$. There will be reductions in the bandwidth due to finite speed of the choppers and the need to guarantee that no neutrons from different pulses can reach the detector while it is counting the frame of interest, but we can calculate the "ideal" TOF Gain for this simulation as a function of neutron wavelength as

$$
G=3 \AA /(0.12 \lambda) \text {. }
$$

Thus for $10-\AA$ neutrons we can expect a gain of nearly 2.5 , and at $6 \AA$ a gain of about 4 . This is quite a bit smaller than the nominal factor of about 15 from duty-cycle arguments, because the resolution required for small-angle scattering is generally worse than can be achieved at a spallation source. Note that if resolution better than $12 \%$ (FWHM) is useful, then the TOF gain factor will be correspondingly larger. 
This simplified estimate of the TOF Gain leaves several "real-life" questions to be answered:

What factors affect the $\Delta \lambda$ bandwidth?

- Is the TOF resolution as good as $\mathrm{CW}$ ?

- Does the full bandwidth contribute useful information?

- What is the dynamic range in Q?

To find the answers to these questions we must include the effects of the neutron pulse shape and the choppers, as well as the geometry of the collimation and detector. Monte Carlo techniques will be used.

\section{Monte Carlo Library MCLIB}

Monte Carlo is a method to integrate over a large number of variables. Random numbers are used to select a value for each variable, and the integrand is evaluated. The process is repeated a large number of times and the resulting values are averaged. For a neutron transport problem, we first select a neutron from the source distribution, and project it through the instrument using either deterministic or probabalistic algorithms to describe its interaction whenever it hits something, and then (if it hits the detector) tally it in a histogram representing where and when it was detected. This is intended to simulate the process of running an actual experiment (but it is much slower).

The present MCLIB library has been derived from codes written by Mike Johnson at the Rutherford Laboratory [3]. Significant additions and revisions were made by this author in 1984 , and the entire code was rewritten in a structured form in 1994. Whenever the code has been applied to new problems, additions have been made. Thus significant contributions to the present library have been made by Richard Heenan (Rutherford-Appleton Laboratory), and specifically for the LPSS by Glenn Olah, Bob VonDreele, Greg Smith, and Luke Daemen (LANSCE). Mike Fitzsimmons and Joyce Goldstone have contributed greatly to the debugging process.

The geometry of a system is described by surfaces and regions. A surface is defined by a general 3-dimensional quadratic equation of the form

$$
A x^{2}+B x+C y^{2}+D y+E z^{2}+F z+G+P x y+Q y z+R z x=0
$$

with 10 coefficients, plus a roughness parameter. The geometric shape of each region is defined by its relationship to each surface: the region is said to be on the positive (negative) side of a surface if the quadratic expression for the surface evaluates to a positive (negative) value for all points within the region, and the entry for that surface in the region structure is therefore made positive (negative). Surfaces which do not bound the region are set to zero in the structure. Information about the surface (e.g., totally reflecting or absorbing) is stored by using numeric values other than \pm 1 for that surface in the region structure. Information about what is contained within a region is stored by assigning a predefined type number (e.g., 31 for a scattering sample of hard spheres) to the region, along with however many parameters are needed. Future development of the library should be accomplished by defining new region types and implementing the corresponding algorithms for how a neutron interacts in such regions.

Features of MCLIB which are different from other Monte Carlo libraries include 
- Simplified transmission through materials. Rather than compute microscopic interaction in a simple (amorphous unpolarized) region, attenuation of the transmitted neutron is calculated.

Optics at surfaces. When a neutron reaches a surface, the (complex) index of refraction is computed to decide whether the neutron will reflect or refract.

- Time-dependent devices. There are region types to describe moving devices such as choppers or a gravity focuser.

- Scattering functions. Each kind of scattering sample is a region type. The scattering algorithm may be deterministic (reflectometry), probabalistic (hard-sphere scatterer), or a combination (Bragg reflection into a Debye-Scherrer cone).

\section{Results of Monte Carlo Simulations}

Since we will be combining and comparing distributions of different shapes, is essential that we define "resolution" in proper statistical terms as the root-mean-square (rms or standard deviation, $\sigma$ ) of a distribution, rather than trying to represent it as full-width-half-maximum. For instance, for a square distribution such as the proton pulse width or a detector pixel, $\sigma$ (square) $=$ FWHM $/ \sqrt{ } 12$; for a triangular distribution such as the velocity selector, $\sigma$ (triangle) $=\mathrm{FWHM} / \sqrt{ } 6$; and for an exponential such as the long-time response of the moderator, $\sigma($ exponential $)=\tau=$ FWHM/ln(2). The $\sigma$ values may always be combined quadratically to calculate the $\sigma$ of a convolution, or it may be computed from the second moment of the result. After many effects are combined, the resulting distribution will approach a Gaussian, so that the standard deviation may be converted back to FWHM using $\sigma($ Gaussian $)=F W H M / \sqrt{ }(8 \ln (2))=F W H M / 2.355$.

Figure 2 illustrates the raw data result of the simulation of D11 (cf. fig. 1), for the velocity selector centered at $10-\AA$, scattering at a fixed value of $Q=0.010 \AA^{-1}$, integrated in rings on a $640-\mathrm{mm}$ square detector with $5 \times 5 \mathrm{~mm}^{2}$ pixels and $\mathrm{rms}$ encoding precision $3.4 \times 3.4 \mathrm{~mm}^{2}$. The peak is at $170 \mathrm{~mm}$ radius, and double scattering gives a second peak just off the edge of the detector, but visible in the corners. When converted to $\mathrm{Q}$, both the accuracy and precision of the result are essentially perfect, and the resolution is $\sigma=10.7 \%$. This test simulation must now be compared to the LPSS case.

The moderator spectrum and also the time distribution of neutron emission (which is a function of neutron wavelength) are important when simulating a spallation source. We have used the spectrum for the coupled $\mathrm{H}_{2}$ moderator, with Be reflector decoupled at a radius $60 \mathrm{~cm}$, as presented by Gary Russell [4]. The time dependence from the output of the MCNP computation has been fitted to a 6-parameter algorithm. We now try to answer the "real life" questions asked above.

What factors affect the $\Delta \lambda$ bandwidth?

As indicated in fig. 1 and in the distance-time diagram in fig. 3, there are three choppers in the LPSS simulation. The first, placed at $5.2 \mathrm{~m}$ from the moderator, is a massive " $\mathrm{T}_{0}$ " chopper to reduce room background for the duration of the proton pulse. The second chopper ("frame overlap", at $5.8 \mathrm{~m}$ ) assures that fast neutrons from the following pulse cannot reach the detector. The actual "frame definition" chopper has been placed at $9.2 \mathrm{~m}$ (based on an optimization study by Glenn Olah). Its phase is adjusted to be half closed for the maximum wavelength to be 
recorded for neutrons emitted at $t=0$; this defines Tmax. The chopper must not begin to open again until the tail of the following pulse has decayed to $1 / 1000$. Finally, the Tmin of the following pulse is defined by the delay after Tmax after which we can guarantee that the frame definition chopper is fully closed (including phase jitter). As shown in fig. 3, there is a large penumbra associated with the finite velocity and the phase jitter of the choppers, and the bandwidth is limited by the overlap between closing for one pulse and opening for the next. These effects are seen quantitatively in fig. 4 , which shows two Monte Carlo results for neutron spectra passed by the choppers when set for bands centered at $6 \AA$ and $10 \AA$ respectively.

\section{- Is the TOF resolution as good as CW?}

The most significant time-of-emission parameter for the relatively long wavelength neutrons used here is the exponential decay of the Maxwellian component, which is $375 \mu \mathrm{s}$. This exponential dominates the time resolution; when it is convoluted with the $1 \mathrm{~ms}$ wide proton pulse, the resulting time resolution is $473 \mu \mathrm{s}$, corresponding to $0.09 \AA$ at a flight path of $21 \mathrm{~m}$. This is better than the $4.9 \%$ (rms) resolution of the Costanze velocity selector at any wavelength longer than $2 \AA$. In the LPSS simulation near $10 \AA$, data were tallied in 29 nominal wavelength slices depending on time of arrival at the detector. Thus we acquired 29 data sets similar to fig. 2 ; fig. 5 presents these on a 2-dimensional plot. For each longer wavelength, the scattered peak is further out on the detector. The velocity selector corresponds to adding up the center 12 rows, with a consequent degradation of resolution. TOF gives us the power to make wavelengthdependent corrections, and to convert each slice to a function of $\mathrm{Q}$ before combining, and thus to retain the full resolution. TOF resolution is inherently better than a velocity selector.

- Does the full bandwidth contribute useful information?

For the case shown in fig. 5 , the answer is "yes". For the case near $6 \AA$, however, a good deal of the count rate comes from neutrons nearer to $5 \AA$ wavelength. The peak for those slices is at $5 / 6$ of the detector radius, and the relative error due to detector geometry increases by $6 / 5$. To keep resolution equal to that of D11, the first 12 or so (of 48 ) time slices would be omitted from the final data analysis. This is indicated in Table 1 in the row labeled "Equal Resolution." The user may later decide to sacrifice resolution for higher count rate; this gives the "Maximum Count Rate" row in Table 1. The table shows what fraction of our original estimate of "Ideal" TOF Gain is realized in a relatively realistic simulation of $\mathrm{D} 11$.

- What is the dynamic range in $\mathrm{Q}$ ?

From fig. 4 we see that useful data may be obtained at wavelengths both longer and shorter than the nominal band center. The effect is limited by the pulse repetition rate, however, and is not very significant at $60 \mathrm{~Hz}$. Most experiments would require two wavelength-band settings, which involve changing the phasing of the choppers. Another option would be to have the phase of the frame overlap and frame definition choppers continuously varying (e.g., by running at $59.9 \mathrm{~Hz}$ instead of $60 \mathrm{~Hz}$ ), so that every 10 seconds or so the complete $\mathrm{Q}$ range would be collected. This could be helpful in measuring kinetics of systems on time scales of minutes or shorter.

\section{Conclusions}

Table 2 compares the two simulated experimental results on a D1 1-type instrument at a 1-MW LPSS to the identical instrument on the ILL reactor, using $6-\AA$ and $10-\AA$ neutrons, respectively. The LPSS exceeds the CW source in all four performance measures: count rate is higher; 
minimum Q is lower; resolution is better; and dynamic range is higher. The latter three of these are not terribly significant, and are readily understood from the use of TOF instead of a velocity selector. We must ask, however, why the count rate is significantly higher than ILL, when we expect our time-average flux to be only a quarter of that of the $60-\mathrm{MW}$ reactor. In fig. 4 we see that our estimated flux at $10 \AA$ is $63 \%$ of the peak for Costanze, or a factor of about 2.5 higher than anticipated.

We believe there are three possibly significant loss terms in the flux observed at D11. D11 is not on the CS-2 cold source, but on an older source approximately $2 / 3$ as efficient. There are about 80 meters of guide between the source and D11, and if the loss is $1 \%$ per meter then the transmission is about $45 \%$. The transmission of the velocity selector may be $80 \%$. Combining these three worst-case factors would give a factor of 4 decrease of the D11 flux from the ideal CW source. Thus it is relatively easy to account for a factor of 2.5 inefficiency at D11.

Thus we believe the scaling rules work. Furthermore, we state that a D11-type instrument at a 1-MW LPSS would significantly outperform the existing D11. Optimization studies begun by Glenn Olah show that some additional gains (of the order $20-30 \%$ in count rate, and even larger improvements in resolution) are possible beyond those shown in Table 2 , by using a circular collimator entrance aperture and using assymetric flight paths. We also note that these results depend strongly on the pulse rate; at $120 \mathrm{~Hz}$ instead of $60 \mathrm{~Hz}$, for example, we would have half the TOF Gain and would only be equivalent to D 11 instead of double.

\section{References,}

1. P. Lindner, R. P. May, and P. A. Timmins, "Upgrading of the SANS instrument D11 at the ILL," Physica B180 (1992) 967.

2. P. Lindner, e-mail communication, March 19, 1995.

3. M. W. Johnson and C. Stephanou, "MCLIB: A Library of Monte Carlo Subroutines for Neutron Scattering Problems," Rutherford Laboratory report RL-78-090.

4. G. J. Russell, "The Calculated Performance of a Benchmark Target/Moderator System for an LPSS," these proceedings. 
Table 1: Time-Of-Flight Gain Factor

3-A Bandwidth vs.

$12 \%$ Velocity Selector

\begin{tabular}{c|c|c} 
& $\lambda=6 \AA$ & $\lambda=10 \AA$ \\
\hline $\begin{array}{c}\text { "Ideal" } \\
\text { TOF Gain }\end{array}$ & 4.2 & 2.5 \\
\hline $\begin{array}{c}\text { "Real Life" } \\
\text { Equal } \\
\text { Resolution }\end{array}$ & 2.7 & 2.2 \\
\hline $\begin{array}{c}\text { "Real Life" } \\
\text { Maximum } \\
\text { Count Rate }\end{array}$ & 3.8 & 2.2
\end{tabular}

Table 2: Calculated Ratios of D11 Monte Carlo Benchmark

at $Q=0.01 \mathrm{~A}^{-1}$

1-MW LPSS / ILL

\begin{tabular}{c|c|c} 
& $\lambda=6 \AA$ & $\lambda=10 \AA$ \\
\hline $\begin{array}{c}\text { Count Rate: } \\
\text { high resolution } \\
\text { (total) }\end{array}$ & $\begin{array}{c}2.8 \\
(4.0)\end{array}$ & 1.4 \\
\hline$Q_{\min }$ & 0.80 & 0.88 \\
\hline $\begin{array}{c}\text { Q Resolution: } \\
\text { at reduced rate } \\
\text { (full count rate) }\end{array}$ & $\begin{array}{c}0.95 \\
(1.10)\end{array}$ \\
\hline & 1.3 & 0.93 \\
\hline$Q_{\max }$ & & 1.2
\end{tabular}




\section{Small-Angle Scattering Simulations}

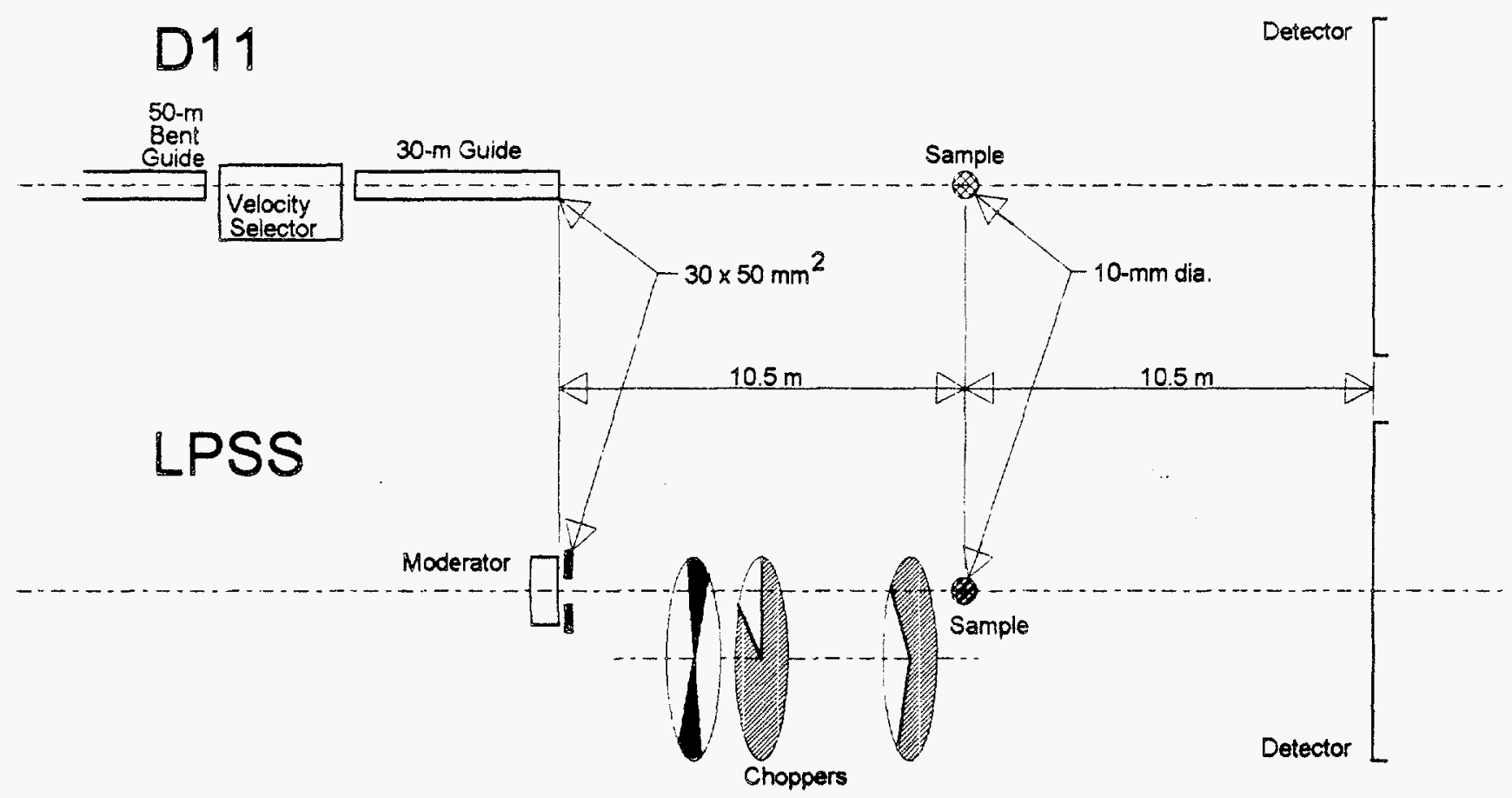

Figure 1. Geometry of the test case for comparing small-angle neutron scattering at a $\mathrm{CW}$ source $v s$. an LPSS. Identical collimation, sample, and detectors are used to assure identical geometric contributions to the resolution. 


\section{Radial Integration of 2-Dimensional Detector}

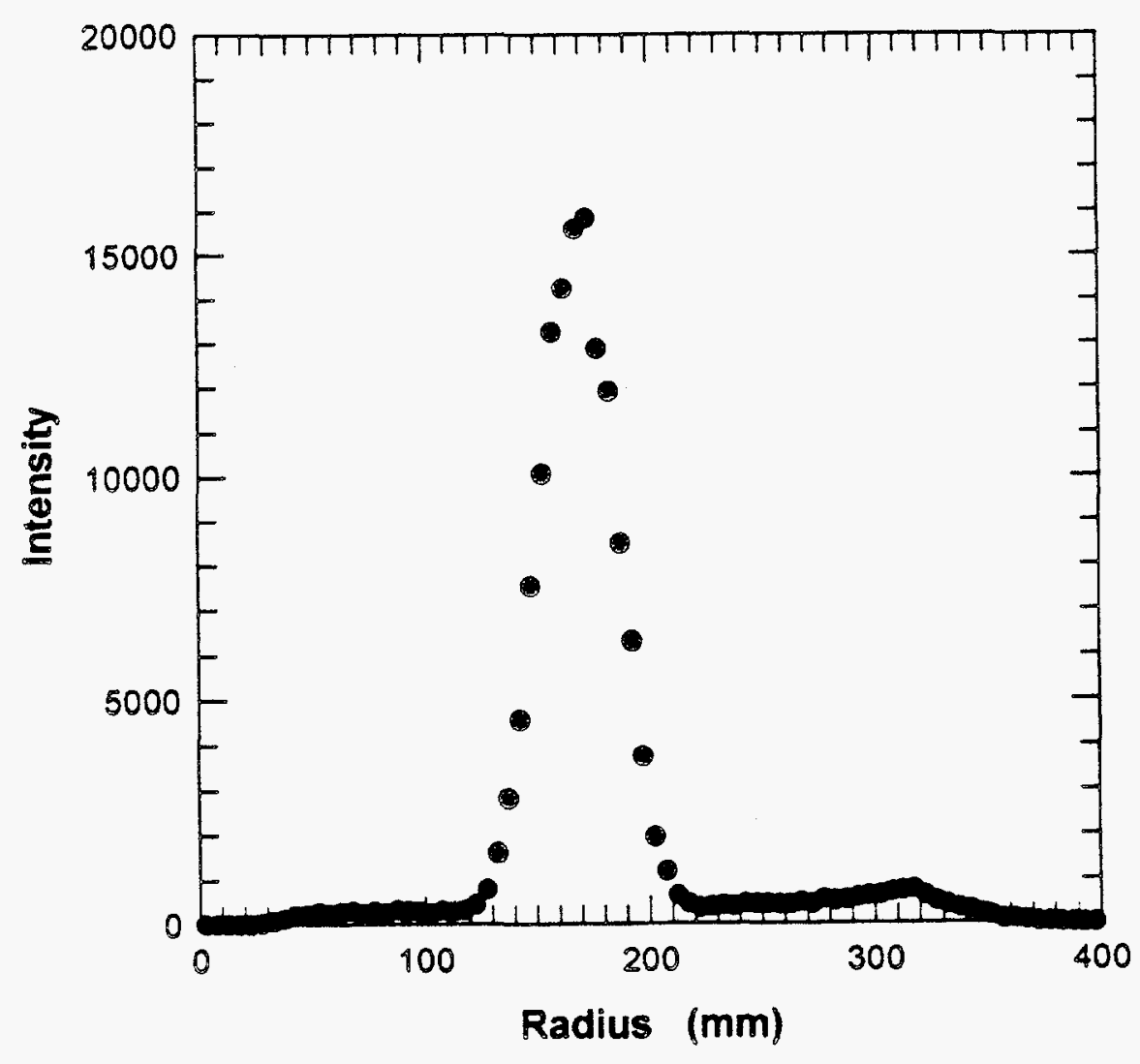

Figure 2. Simulated response of D11 (at ILL) for a hypothetical scattering sample which scatters every neutron at the same value of $Q\left(Q=0.01 \AA^{-1}\right)$. The shape of the peak is the overall resolution of the instrument, including both geometric and wavelength contributions. Double scattering can also be seen. 


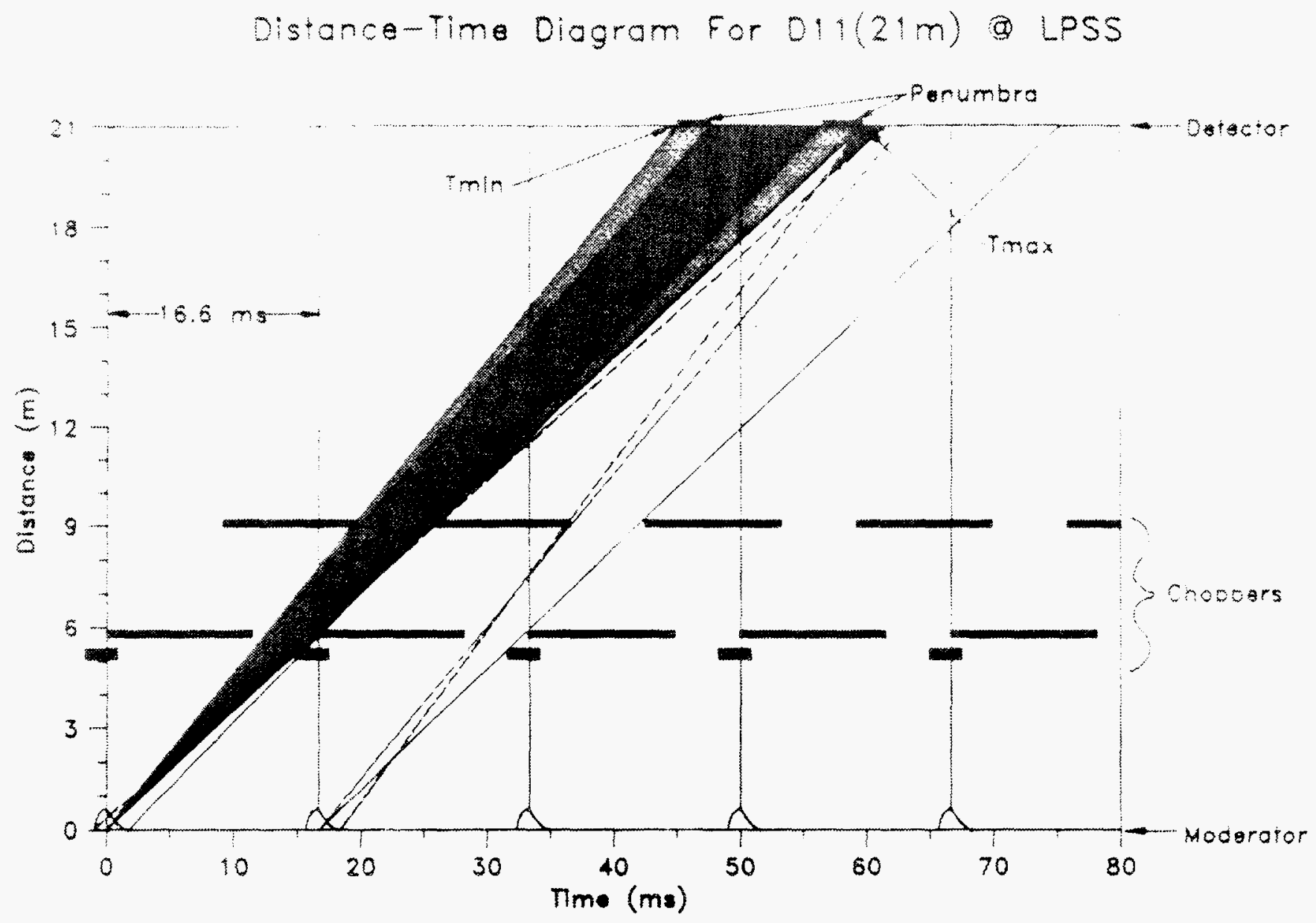

Figure 3. Chopper phasing diagram. The functions of the three choppers are defined in the text. Slopes of the lines are equal to neutron velocity, which is inversely proportional to wavelength. With the phases drawn here, neutrons of wavelengths 8.5 to $11.5 \AA$ are being counted in the detector. 


\section{Spectrum Comparison at $6 \mathrm{~A}$ and $10 \mathrm{~A}$}

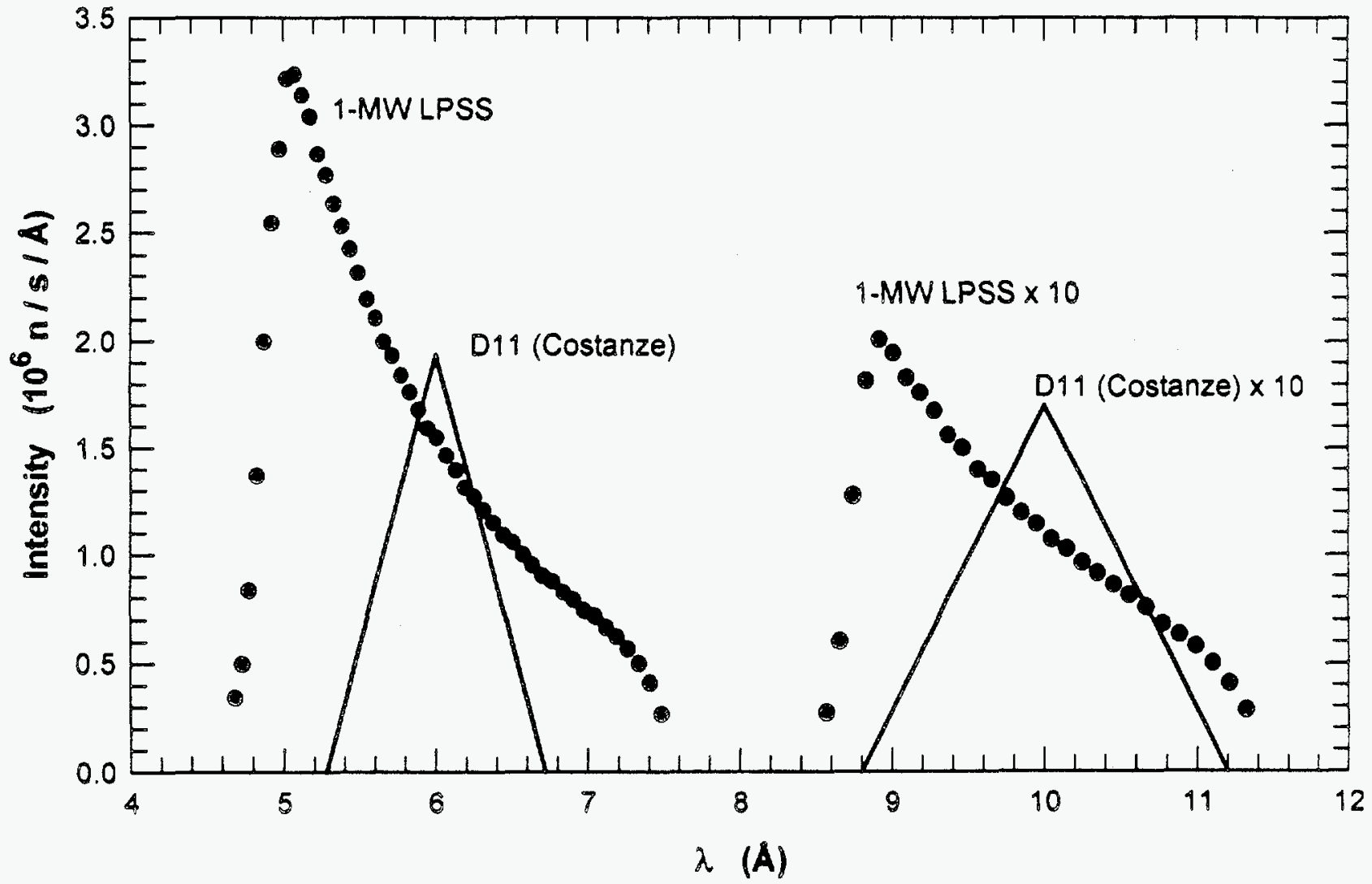

Figure 4. Neutron spectra passed by the chopper system at two different phase settings, compared to the assumed triangular distribution of the "Costanze" velocity selector. 


\section{Normalized Monte Carlo Data \\ $11 \mathrm{~m}, 21 \mathrm{~m}, 11 \mathrm{~A}, 60 \mathrm{~Hz}(1000 \mu \mathrm{s}), \mathrm{Q}=0.01 / \mathrm{A}$ \\ (logarithmic contour intervals)}

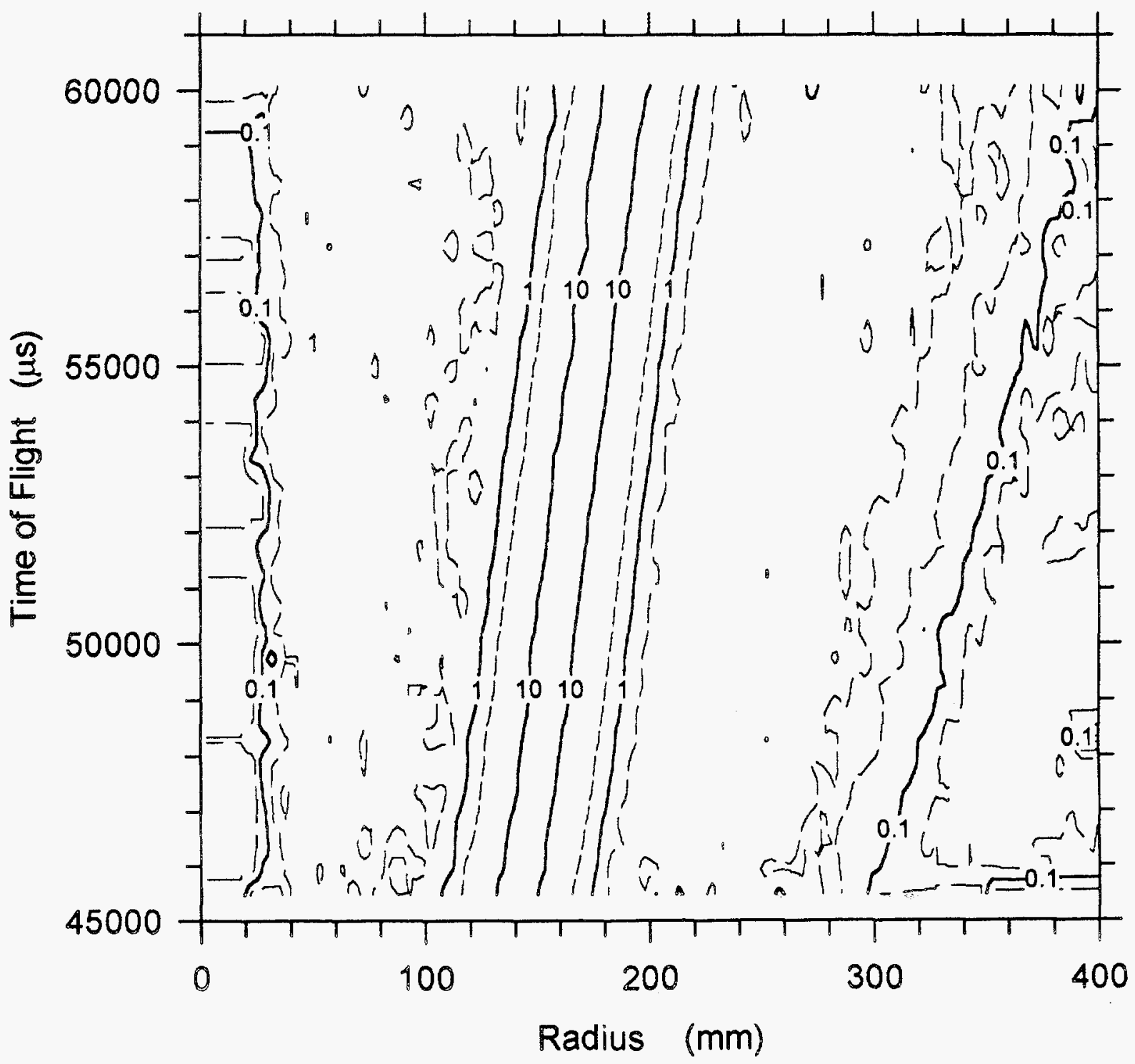

Figure 5. Radially integrated data for the 1-MW LPSS simulation. A horizontal slice would correspond to a single wavelength, and would be similar to fig. 2 . The 29 independent wavelength slices are converted to a $\mathrm{Q}$ scale before summing, virtually eliminating the wavelength contribution to the resolution. 